\title{
Type II Bivariate Generalized Power Series Poisson Distribution and its Applications in Risk Analysis
}

\author{
K.K Jose ${ }^{\mathrm{a}, *}$ and Shalitha Jacob ${ }^{\mathrm{a}, \mathrm{b}}$ \\ ${ }^{a}$ Department of Statistics, St. Thomas College, Pala, Arunapuram, Kerala-686574, India \\ ${ }^{b}$ Mar Thoma College for Women, Perumbavoor, Kerala-683542, India
}

\begin{abstract}
In this paper we consider type II bivariate generalized power series Poisson distribution as a compound Poisson distribution with bivariate generalized power series compounding distribution. We obtain some properties, p.m.f and conditional distributions. In addition we also give a brief discussion about the multivariate extension of this case. Then we introduce type II bivariate generalized power series Poisson process and consider a bivariate risk model with type II bivariate generalized power series Poisson model as the counting process. For this model we derive distribution of the time to ruin and bounds for the probability of ruin. We obtain partial integro-differential equation for the ruin probabilities and express its bivariate transform through two univariate boundary transforms, where one of the initial capitals is fixed at zero.
\end{abstract}

Keywords: bivariate generalized power series distribution; ruin probability; aggregate claims distribution

\section{Introduction}

Bivariate discrete random variables taking non-negative integer values, have received considerable attention in the literature. The type II bivariate Polya - Aeppli distribution was introduced by Minkova and Balakrishnan(2014). Kostadinova and Minkova(2014) applied bivariate Poisson negative binomial distribution to bivariate risk processes.

5 Furthermore Kostadinova(2015) introduced a bivariate risk model in which distribution of claim counting process is the bivariate Polya-Aeppli distribution. In the literature it has been found that bivariate compound Poisson distributions are very flexible and can be used efficiently in bivariate risk modeling. With this as motivation, different bivariate compound Poisson distributions have been constructed.

The family of bivariate generalized power series distribution is basically used for counting paired events. It contains many important families like bivariate Poisson, bivariate binomial, bivariate negative binomial and bivariate

\footnotetext{
${ }^{*}$ Corresponding author

Email address: kkjstc@gmail.com (K.K Jose)

$U R L$ : shalithajacob@yahoo.in (Shalitha Jacob)
} 
logarithmic series distributions. The PMF of bivariate generalized power series distribution is given by

$$
P(i, j)=\frac{a_{i, j} \theta_{1}^{i} \theta_{2}^{j}}{g\left(\theta_{1}, \theta_{2}\right)}, \quad(i, j) \in \mathcal{T}
$$

where $g\left(\theta_{1}, \theta_{2}\right)=\sum_{i, j} a_{i, j} \theta_{1}^{i} \theta_{2}^{j}, \quad a_{i, j} \geq 0$ and $\mathcal{T}$ is a subset of cartesian product of the set of nonnegative integers.

In this paper we consider compound Poisson distribution with bivariate generalized power series compounding distribution. The bivariate type II Polya-Aeppli distribution and the bivariate Poisson negative binomial distribution are the special cases of bivariate generalized power series Poisson distribution.

For the univariate case, where $X_{1}, X_{2}, X_{3} \ldots$ are independent and identically distributed random variables, independent of $N_{1}$ and $N_{1}$ has a Poisson distribution with parameter $\lambda$, denoted by $N_{1} \sim P_{o}(\lambda)$. Suppose that $X_{1}, X_{2}, X_{3} \ldots$ follow generalized power series distribution with PGF

$$
P(s)=\frac{g(\theta s)}{g(\theta)}
$$

where $g(\theta)=\sum_{i} a_{i} \theta^{i}, \quad a_{i} \geq 0$.

Now consider the random sum

$$
N=X_{1}+X_{2}+\ldots+X_{N_{1}}
$$

The distribution of $N$ is called generalized power series Poisson distribution.

The PGF of the random variable $N$ is given by

$$
\Psi_{N}(s)=e^{-\lambda(1-P(s))}=e^{-\lambda\left(1-\frac{g(\theta s)}{g(\theta)}\right)}
$$

Then the corresponding PMF is given by

$$
\begin{aligned}
P(N=m) & =e^{-\lambda}, \quad m=0 \\
& =e^{-\lambda} \theta^{m} \sum_{j=1}^{\infty} \frac{C_{m}(j)\left(\frac{\lambda}{g(\theta)}\right)^{j}}{j !}, \quad m=1,2, \ldots
\end{aligned}
$$

where

$$
C_{m}(j)=\sum_{k_{1}+k_{2}+\ldots+k_{j}=m} a\left(k_{1}\right), a\left(k_{2}\right) \ldots a\left(k_{j}\right), \quad \text { If }\left(k_{1}, k_{2}, \ldots, k_{j}\right) \text { is the ordered j-tuple of }
$$

positive integers in the range set of the random variable $\mathrm{X}$ which sum up to $\mathrm{m}$.

This paper is organised as follows. In section 2 the joint probability mass function, correlation coefficient and covariance of type II bivariate generalized power series Poisson distribution are derived. In section 3 marginal distribution and conditional distribution of type II bivariate generalized power series Poisson distribution are given. 
A multivariate extension of the generalized power series Poisson distribution and its properties are discussed in section 4. Bivariate counting processes with type II bivariate generalized power series Poisson distribution is introduced in section 5. In section 6 we consider type II bivariate generalized power series Poisson risk model and 25 derive the distributions of bivariate aggregate claims and sum of aggregate claims of two classes. Section 7 presents three types of ruin probabilities and an expression for ruin probabilities for a type II bivariate generalized power series Poisson risk model is derived. In addition, the bounds for the ruin probabilities are developed. In section 8 a system of partial integro differential equation for the ruin probabilities is developed and the Laplace transform is derived. Section 8 deals with multivariate generalized power series Poisson risk model and the ruin probabilities for the defined risk model.

\section{Bivariate Generalized Power Series Poisson Distribution}

Let us consider the sequence $\left(X_{i}, Y_{i}\right), i=1,2, \ldots$ of independent and identically distributed random variables, distributed as $(X, Y)$.

Define

$$
N_{1}=X_{1}+X_{2}+\ldots+X_{N} \text { and } N_{2}=Y_{1}+Y_{2}+\ldots+Y_{N}
$$

where $N$ is independent of the compounding random vector $(X, Y)$ and has a Poisson distribution with parameter $\lambda$.

Suppose that $(X, Y)$ has a bivariate generalized power series distribution with PGF

$$
P\left(s_{1}, s_{2}\right)=\frac{g\left(\theta_{1} s_{1}, \theta_{2} s_{2}\right)}{g\left(\theta_{1}, \theta_{2}\right)} .
$$

Then, the joint PGF of the bivariate random vector $\left(N_{1}, N_{2}\right)$ is given by

$$
\Psi\left(s_{1}, s_{2}\right)=e^{-\lambda\left(1-P\left(s_{1}, s_{2}\right)\right)}=e^{-\lambda\left(1-\frac{g\left(\theta_{1} s_{1}, \theta_{2} s_{2}\right)}{g\left(\theta_{1}, \theta_{2}\right)}\right)}
$$

The PGF in 2) can be written as

$$
\Psi\left(s_{1}, s_{2}\right)=e^{-\lambda} \sum_{k=1}^{\infty} \frac{\left(\frac{\lambda}{g\left(\theta_{1}, \theta_{2}\right)}\right)^{k}}{k !} \sum_{i, j} C_{i j}(k)\left(\theta_{1} s_{1}\right)^{i}\left(\theta_{2} s_{2}\right)^{j}
$$

40 where

$C_{i j}(k)=\sum_{\substack{i_{1}+i_{2}+\cdots+i_{k}=i \\ j_{1}+j_{2}+\cdots+j_{k}=j}} a_{i_{1} j_{1}} a_{i_{2} j_{2}} \cdots a_{i_{k} j_{k}}$, if $\left(i_{1}, i_{2}, \cdots, i_{k}\right)$ is the ordered $k$ tuple of elements in the range of $X$ which sum up to $i$ and $\left(j_{1}, j_{2}, \cdots j_{k}\right)$ is the ordered $k$ tuple of elements in the range of $Y$ which sum up to $j$. 
Differentiation in (3) leads to the following derivatives

$$
\Psi^{(i, j)}\left(s_{1}, s_{2}\right)=e^{-\lambda} \sum_{k=1}^{\infty} \frac{\left(\frac{\lambda}{g\left(\theta_{1}, \theta_{2}\right)}\right)^{k}}{k !} \sum_{l \geq i, m \geq j} C_{l, m}(k) l^{(i)} m^{(j)} \theta_{1}^{l} \theta_{2}^{m} s_{1}^{l-i} s_{2}^{m-j},
$$

where $l^{(i)}=\frac{l !}{(l-i) !}, m^{(j)}=\frac{m !}{(m-j) !}$ and $\Psi^{(i, j)}\left(s_{1}, s_{2}\right)=\frac{\partial^{i+j}}{\partial s_{1}^{i} \partial s_{2}^{j}} \Psi\left(s_{1}, s_{2}\right)$.

Setting $s_{1}=s_{2}=1$ in (4), we obtain the $(i, j)^{t h}$ factorial moments of $\left(N_{1}, N_{2}\right)$

$$
E N_{1}\left(N_{1}-1\right) \cdots\left(N_{1}-i+1\right) N_{2}\left(N_{2}-1\right) \cdots\left(N_{2}-j+1\right)
$$

$$
=e^{-\lambda} \sum_{k=0}^{\infty} \frac{\left(\frac{\lambda}{g\left(\theta_{1}, \theta_{2}\right)}\right)^{k}}{k !} \sum_{l \geq i, m \geq j} C_{l m}(k) l^{(i)} m^{(j)} \theta_{1}^{l} \theta_{2}^{m}
$$

\subsection{Covariance and Correlation}

The means are given by

$\mu_{1}=E\left(N_{1}\right)=\lambda \theta_{1} \frac{\partial}{\partial \theta_{1}} \log g\left(\theta_{1}, \theta_{2}\right)$ and $\mu_{2}=E\left(N_{2}\right)=\lambda \theta_{2} \frac{\partial}{\partial \theta_{2}} \log g\left(\theta_{1}, \theta_{2}\right)$.

Similarly the variances are obtained as

$\operatorname{Var}\left(N_{1}\right)=\theta_{1} \frac{\partial}{\partial \theta_{1}} \mu_{1}+\frac{1}{\lambda} \mu_{1}^{2}, \operatorname{Var}\left(N_{2}\right)=\theta_{2} \frac{\partial}{\partial \theta_{2}} \mu_{2}+\frac{1}{\lambda} \mu_{2}^{2}$.

From (2), we obtain

$$
\begin{gathered}
\frac{\partial^{2}}{\partial s_{1} \partial s_{2}} \Psi\left(s_{1}, s_{2}\right)=\left(\frac{\lambda}{g\left(\theta_{1}, \theta_{2}\right)} \frac{\partial^{2}}{\partial s_{1} \partial s_{2}} g\left(\theta_{1} s_{1}, \theta_{2} s_{2}\right)+\left(\frac{\lambda}{g\left(\theta_{1}, \theta_{2}\right)}\right)^{2} \frac{\partial}{\partial s_{1}} g\left(\theta_{1} s_{1}, \theta_{2} s_{2}\right)\right. \\
\left.\frac{\partial}{\partial s_{2}} g\left(\theta_{1} s_{1}, \theta_{2} s_{2}\right)\right) \Psi\left(s_{1}, s_{2}\right)
\end{gathered}
$$

Setting $s_{1}=s_{2}=1$ in $(5)$, we easily obtain

$$
E N_{1} N_{2}=\theta_{2} \frac{\partial}{\partial \theta_{2}} \mu_{1}+\left(1+\frac{1}{\lambda}\right) \mu_{1} \mu_{2}
$$

The covariance and correlation between $N_{1}$ and $N_{2}$ are respectively given by

$$
\begin{aligned}
\operatorname{Cov}\left(N_{1}, N_{2}\right) & =\theta_{2} \frac{\partial}{\partial \theta_{2}} \mu_{1}+\frac{1}{\lambda} \mu_{1} \mu_{2} \\
\operatorname{Corr}\left(N_{1}, N_{2}\right) & =\frac{\theta_{2} \frac{\partial}{\partial \theta_{2}} \mu_{1}+\frac{1}{\lambda} \mu_{1} \mu_{2}}{\sqrt{\prod_{i=1}^{2}\left(\theta_{i} \frac{\partial}{\partial \theta_{i}} \mu_{i}+\frac{1}{\lambda} \mu_{i}^{2}\right)}} .
\end{aligned}
$$

50 2.2. Joint Probability Mass Function

The joint probability mass function of $\left(N_{1}, N_{2}\right)$ is obtained by expanding the PGF, $\Psi\left(s_{1}, s_{2}\right)$ in powers of $s_{1}$ and $s_{2}$.

Let $f(i, j)=P\left(N_{1}=i, N_{2}=j\right), i=j=0,1,2 \ldots$ denote the joint PMF of $\left(N_{1}, N_{2}\right)$.

On the other hand, from Johnson et al., it is known that

$$
f(i, j)=\frac{\Psi^{(i, j)}\left(s_{1}, s_{2}\right)}{i ! j !} / s_{1}=s_{2}=0 .
$$


Using the PGF in (2) and the derivatives in (4) we obtain the joint PMF of $\left(N_{1}, N_{2}\right)$ and is given by

$$
\begin{aligned}
f(0,0) & =e^{-\lambda\left(1-\frac{a_{0,0}}{g\left(\theta_{1}, \theta_{2}\right)}\right)}, \\
f(i, j) & =e^{-\lambda} \theta_{1}^{i} \theta_{2}^{j} \sum_{k=1}^{\infty} \frac{C_{i j}(k)\left(\frac{\lambda}{g\left(\theta_{1}, \theta_{2}\right)}\right)^{k}}{k !}, i, j=0,1, \cdots,(i, j) \neq(0,0),
\end{aligned}
$$

where $C_{i j}(k)=\sum_{\substack{i_{1}+i_{2}+\cdots+i_{k}=i \\ j_{1}+j_{2}+\cdots+j_{k}=j}} a_{i_{1}, j_{1}} a_{i_{2}, j_{2}} \cdots a_{i_{k}, j_{k}}$, if $\left(i_{1}, i_{2}, \cdots, i_{k}\right)$ is the ordered $k$ tuple of elements in the range of $X$ which sums up to $i$ and $\left(j_{1}, j_{2}, \cdots j_{k}\right)$ is the ordered $k$ tuple of elements in the range of $Y$ which sums up to $j$

\section{Marginal Distributions}

The PGFs of the marginal compounding distributions are given by

$P_{1}\left(s_{1}\right)=P\left(s_{1}, 1\right)=\frac{g\left(\theta_{1} s_{1}, \theta_{2}\right)}{g\left(\theta_{1}, \theta_{2}\right)}=\frac{\sum_{i} b_{i}\left(\theta_{1} s_{1}\right)^{i}}{\sum_{i} b_{i} \theta_{1}^{i}}$, where $b_{i}=\sum_{j} a_{i j} \theta_{2}^{j}$, is independent of $\theta_{1}$, $P_{2}\left(s_{2}\right)=P\left(1, s_{2}\right)=\frac{g\left(\theta_{1}, \theta_{2} s_{2}\right)}{g\left(\theta_{1}, \theta_{2}\right)}=\frac{\sum_{j} c_{j}\left(\theta_{2} s_{2}\right)^{j}}{\sum_{j} c_{j} \theta_{2}^{j}}$, where $c_{j}=\sum_{i} a_{i j} \theta_{1}^{i}$, is independent of $\theta_{2}$.

Therefore the above marginal PGFs can be written in the form

$$
\begin{aligned}
& P_{1}\left(s_{1}\right)=\frac{h_{1}\left(\theta_{1} s_{1}\right)}{h_{1}\left(\theta_{1}\right)} \\
& P_{2}\left(s_{2}\right)=\frac{h_{2}\left(\theta_{2} s_{2}\right)}{h_{2}\left(\theta_{2}\right)} .
\end{aligned}
$$

From which it follows that the random variable $\mathrm{X}$ has a generalized power series distribution with series function $h_{1}\left(\theta_{1}\right)=\sum_{i} b_{i} \theta_{1}^{i}$, where $b_{i}=\sum_{j} a_{i j} \theta_{2}^{j}$ and $\theta_{2}$ are treated as constants.

Analogously, Y has a generalized power series distribution with series function $h_{2}\left(\theta_{2}\right)=\sum_{j} c_{j} \theta_{2}^{j}$, where $c_{j}=$ $\sum_{i} a_{i j} \theta_{1}^{i}$ and $\theta_{1}$ are treated as constants.

Then , from (2) and (6), we obtain the corresponding marginal PGFs of $N_{1}$ and $N_{2}$

$$
\begin{aligned}
& \Psi_{N_{1}}\left(s_{1}\right)=\Psi\left(s_{1}, 1\right)=e^{-\lambda\left(1-h_{1}\left(\theta_{1}\right)\right)}, \\
& \Psi_{N_{2}}\left(s_{2}\right)=\Psi\left(1, s_{2}\right)=e^{-\lambda\left(1-h_{2}\left(\theta_{2}\right)\right)} .
\end{aligned}
$$

The corresponding marginal distributions of $N_{1}$ and $N_{2}$ are easily obtained from (7), respectively, to be

$$
\begin{aligned}
P\left(N_{1}=m\right) & =e^{-\lambda}, \quad m=0 \\
& =e^{-\lambda} \theta_{1}^{m} \sum_{j=1}^{\infty} \frac{C_{m}(j)\left(\frac{\lambda}{h_{1}\left(\theta_{1}\right)}\right)^{j}}{j !}, \quad m=1,2, \ldots
\end{aligned}
$$

where

$$
C_{m}(j)=\sum_{k_{1}+k_{2}+\ldots+k_{j}=m} b_{k_{1}}, b_{k_{2}} \ldots b_{k_{j}}, \quad \text { If }\left(k_{1}, k_{2}, \ldots, k_{j}\right) \text { is the ordered j-tuple of }
$$

positive integers in the range set of the random variable $\mathrm{X}$ which sum up to $\mathrm{m}$. 
and

$$
\begin{aligned}
P\left(N_{2}=m\right) & =e^{-\lambda}, \quad m=0 \\
& =e^{-\lambda} \theta_{2}^{m} \sum_{j=1}^{\infty} \frac{D_{m}(j)\left(\frac{\lambda}{h_{2}\left(\theta_{2}\right)}\right)^{j}}{j !}, \quad m=1,2, \ldots,
\end{aligned}
$$

where

$$
D_{m}(j)=\sum_{k_{1}+k_{2}+\ldots+k_{j}=m} c_{k_{1}}, c_{k_{2}} \ldots c_{k_{j}}, \quad \text { If }\left(k_{1}, k_{2}, \ldots, k_{j}\right) \text { is the ordered j-tuple of }
$$

positive integers in the range set of the random variable $\mathrm{Y}$ which sum up to $\mathrm{m}$.

${ }_{55}$ Then from it follows that marginal distributions of $N_{1}$ and $N_{2}$ belongs to univariate generalized power series Poisson distribution.

\subsection{Conditional Distribution}

From Johnson et al.(1997), the conditional P.G.F. of $N_{2}$ given $N_{1}$ written $\Psi_{N_{2} / N_{1}=k}\left(s_{2}\right)$, is

$$
\Psi_{N_{2} / N_{1}=k}(s)=\frac{\Psi^{(k, 0)}\left(0, s_{2}\right)}{\Psi^{(k, 0)}(0,1)},
$$

where $\Psi^{(k, 0)}\left(s_{1}, s_{2}\right)=\frac{\partial^{k}}{\partial s_{1}^{k}} \Psi\left(s_{1}, s_{2}\right)$.

Substituting $(i, j)=(k, 0)$ and $s_{1}=0$ in 4 , we get

$$
\Psi^{(k, 0)}\left(0, s_{2}\right)=e^{-\lambda} \sum_{m=1}^{\infty} \sum_{j} \frac{\left(\frac{\lambda}{g\left(\theta_{1}, \theta_{2}\right)}\right)^{m}}{m !} C_{k j}(m) k ! \theta_{1}^{k}\left(\theta_{2} s_{2}\right)^{j} .
$$

60 Using (8) and (9) we obtain

$$
\Psi_{N_{2} / N_{1}=k}\left(s_{2}\right)=\frac{\sum_{m=0}^{\infty} \sum_{j} \frac{\left(\frac{\lambda}{g\left(\theta_{1}, \theta_{2}\right)}\right)^{m}}{m !} C_{k j}(m) \theta_{1}^{k}\left(\theta_{2} s_{2}\right)^{j}}{\sum_{m=1}^{\infty} \sum_{j} \frac{\left(\frac{\lambda}{g\left(\theta_{1}, \theta_{2}\right)}\right)^{m}}{m !} C_{k j}(m) \theta_{1}^{k} \theta_{2}^{j}} .
$$

For $k=0$, we get

$$
\begin{aligned}
\Psi_{N_{2} / N_{1}=0}\left(s_{2}\right) & =\frac{\Psi\left(0, s_{2}\right)}{\Psi(0,1)} \\
& =e^{-\lambda \frac{g\left(0, \theta_{2}\right)}{g\left(\theta_{1}, \theta_{2}\right)}}\left[1-\frac{g\left(0, \theta_{2} s_{2}\right)}{g\left(\theta_{1}, \theta_{2}\right)}\right]
\end{aligned}
$$

It follows immediately that the conditional mean is

$$
E\left[N_{2} / N_{1}=k\right]=\frac{\sum_{m=1}^{\infty} \sum \frac{\left(\frac{\lambda}{f\left(\theta_{1}, \theta_{2}\right)}\right)^{m}}{m !} C_{k j}(m) j \theta_{2}^{j}}{\sum_{m=1}^{\infty} \sum \frac{\left(\frac{\lambda}{f\left(\theta_{1}, \theta_{2}\right)}\right)^{m}}{m !} C_{k j}(m) \theta_{2}^{j}}
$$

In particular

$$
E\left(N_{2} / N_{1}=0\right)=\lambda t \theta_{2} \frac{\frac{\partial}{\partial \theta_{2}} g\left(0, \theta_{2}\right)}{g\left(\theta_{1}, \theta_{2}\right)}
$$




\section{Multivariate Extension}

Let $X=\left(X_{1}, \cdots X_{k}\right)$ be a k-dimensional random vector of generalized power series random variables.

The PGF of $\mathrm{X}$ is given by

$$
P\left(s_{1}, s_{2}, \cdots, s_{k}\right)=\frac{g\left(\theta_{1} s_{1}, \theta_{2} s_{2}, \cdots, \theta_{k} s_{k}\right)}{g\left(\theta_{1}, \theta_{2}, \cdots, \theta_{k}\right)}
$$

Define

$$
N_{i}=X_{i 1}+X_{i 2}+\ldots+X_{i N}, i=1,2, \ldots, k
$$

where $\mathrm{N}$ is independent of compounding random vector $\mathrm{X}$ and has a Poisson distribution with parameter $\lambda$.

Then, the joint PGF of $\left(N_{1}, N_{2}, \cdots, N_{k}\right)$ is given by

$$
\Psi\left(s_{1}, s_{2}, \cdots, s_{k}\right)=e^{-\lambda\left(1-P\left(s_{1}, s_{2}, \cdots s_{k}\right)\right)}=e^{-\lambda\left(1-\frac{g\left(\theta_{1} s_{1}, \theta_{2} s_{2}, \cdots, \theta_{k} s_{k}\right)}{g\left(\theta_{1}, \theta_{2}, \cdots, \theta_{k}\right)}\right)} .
$$

65

The PGFs of the marginal compounding distributions are given by

$$
P_{X_{i}}\left(s_{i}\right)=P\left(1, \cdots, s_{i}, \cdots, 1\right)=\frac{g\left(\theta_{1}, \cdots, \theta_{i} s_{i}, \cdots \theta_{k}\right)}{g\left(\theta_{1}, \cdots, \theta_{k}\right)}=\frac{h_{i}\left(\theta_{i} s_{i}\right)}{h_{i}\left(\theta_{i}\right)}, i=1,2, \cdots k
$$

Where $h_{i}\left(\theta_{i}\right)=\sum_{x i} b_{i}\left(x_{i}\right) \theta_{i}^{x_{i}}$ and $b_{i}\left(x_{i}\right)=\sum_{x_{1} \cdots x_{i-1} x_{i+1} \cdots x_{k}} a_{x_{1} x_{2} \cdots x_{k}} \theta_{1}^{x_{1}} \cdots \theta_{i-1}^{x_{i}-1} \theta_{i+1}^{x_{i}+1} \cdots \theta_{k}^{x_{k}}$.

Therefore from 12 it follows that the random variable $X_{i}$ has a generalized power series distribution with series function $h_{i}\left(\theta_{i}\right)=\sum_{x i} b_{i}\left(x_{i}\right) \theta_{i}^{x_{i}}$ as expanded in powers of $\theta_{i}$, other $\theta^{\prime}$ 's treated as constants.

The marginal PGFs of $N_{i}, i=1,2, \ldots k$ are obtained from 11 and $[12$, and are given by

$$
\Psi_{N_{i}}\left(s_{i}\right)=\Psi\left(1, \ldots, s_{i}, \ldots, 1\right)=e^{-\lambda\left(1-\frac{h_{i}\left(\theta_{i} s_{i}\right)}{h_{i}\left(\theta_{i}\right)}\right)}, i=1,2, \ldots, k
$$

Then from it follows that $N_{i}, i=1,2, \ldots k$ belongs to univariate generalized power series Poisson distribution.

The corresponding marginal P.M.Fs are given by

$$
P\left(N_{i}=m\right)= \begin{cases}e^{-\lambda}, & m=0 \\ e^{-\lambda} \theta_{i}^{m} \sum_{j=1}^{\infty} \frac{\left(\frac{\lambda}{h_{i}\left(\theta_{i}\right)}\right)^{j}}{j} C_{i m}(j), & m=1,2, \ldots,\end{cases}
$$

where $C_{i m}(j)=\sum_{k_{1}+k_{2} \ldots+k_{j}=m} b_{i}\left(k_{1}\right) b_{i}\left(k_{2}\right) \ldots b_{i}\left(k_{j}\right)$ If $\left(k_{1}, k_{2}, \ldots, k_{j}\right)$ is ordered $j$-tuple of positive integers in the range set of $X_{i}$ which sum up to $m$.

\subsection{Joint Probability Mass Function}

The P.G.F. in 11 can be written as

$$
\Psi\left(s_{1}, s_{2}, \ldots, s_{k}\right)=e^{-\lambda} \sum_{j=1}^{\infty} \sum_{i_{1}, i_{2}, \ldots i_{k}} \frac{\left(\frac{\lambda}{g\left(\theta_{1}, \theta_{2}, \ldots, \theta_{k}\right)}\right)^{j}}{j !} C_{i_{1}, i_{2}, \ldots i_{k}}(j)\left(\theta_{1} s_{1}\right)^{i_{1}}\left(\theta_{2} s_{2}\right)^{i_{2}} \ldots\left(\theta_{k} s_{k}\right)^{i_{k}}
$$


70 Differentiation in 13 leads to the following derivatives

$$
\Psi^{\left(r_{1}, r_{2}, \ldots, r_{k}\right)}\left(s_{1}, \ldots, s_{k}\right)=e^{-\lambda} \sum_{j=1}^{\infty} \sum_{i_{1} \geq r_{1}, \ldots, i_{k} \geq r_{k}} \frac{\left(\frac{\lambda}{g\left(\theta_{1}, \theta_{2}, \ldots, \theta_{k}\right)}\right)^{j}}{j !} C_{i_{1}, i_{2}, \ldots, i_{k}}(j) i_{1}^{\left(r_{1}\right)} \theta_{1}^{i_{1}} s_{1}^{i_{1}-r_{1}} \ldots i_{k}^{\left(r_{k}\right)} \theta_{k}^{i_{k}} s_{k}^{i_{k}-r_{k}},
$$

where $i_{j}^{\left(r_{j}\right)}=\frac{i_{j} !}{\left(i_{j}-r_{j}\right) !}, j=1,2, \ldots, k$ and $\Psi^{\left(r_{1}, r_{2}, \ldots, r_{k}\right)}\left(s_{1}, \ldots, s_{k}\right)=\frac{\partial^{r_{1}+r_{2}+\cdots+r_{k}}}{\partial s_{1}^{r_{1}} \partial s_{2}^{r_{2}}, \ldots \partial s_{k}^{r_{k}}} \Psi\left(s_{1}, s_{2}, \ldots, s_{k}\right)$. From Johnson et al.(1997), it is known that for $r_{1} \ldots, r_{k}=0,1 \ldots$, and $\left(r_{1}, r_{2}, \ldots, r_{k}\right) \neq(0, \ldots, 0)$,

$$
f\left(r_{1}, r_{2}, \ldots, r_{k}\right)=\frac{\Psi^{\left(r_{1}, r_{2}, \ldots, r_{k}\right)}\left(s_{1}, \ldots, s_{k}\right)}{r_{1} ! \ldots r_{k} !} / s_{1}=\cdots=s_{k}=0 .
$$

Denote by $f\left(i_{1}, \ldots, i_{k}\right)=P\left(N_{1}=i_{1}, \ldots, N_{k}=i_{k}\right), i_{1} \ldots i_{k}=0,1,2, \ldots$ the joint PMF of $\left(N_{1}, N_{2}, \ldots, N_{k}\right)$ and is given by

$$
\begin{aligned}
& f(0, \ldots, 0)=e^{-\lambda\left(1-\frac{a_{0}, \ldots, 0}{g\left(\theta_{1}, \ldots, \theta_{k}\right)}\right)}, \\
& f\left(i_{1}, i_{2}, \ldots, i_{k}\right)=e^{-\lambda} \sum_{j=1}^{\infty} \frac{\left(\frac{\lambda}{g\left(\theta_{1}, \ldots, \theta_{k}\right)}\right)^{j}}{j !} C_{i_{1}, i_{2}, \ldots, i_{k}}(j) \theta_{1}^{i_{1}} \theta_{2}^{i_{2}} \ldots \theta_{k}^{i_{k}}, \\
& i_{1}, i_{2}, \ldots, i_{k}=0,1, \ldots,\left(i_{1}, i_{2}, \ldots, i_{k}\right) \neq(0,0, \ldots 0),
\end{aligned}
$$

where

$$
\begin{gathered}
C_{i_{1}, i_{2}, \ldots, i_{k}}(j) \sum_{\substack{i_{11}+i_{12},+\ldots,+i_{i j}=i_{1} \\
\vdots \\
i_{k 1}+i_{k 2}+\cdots+i_{k j}=i_{k}}} a\left(i_{11}, i_{21}, \ldots, i_{k 1}\right) a\left(i_{12}, i_{22}, \ldots, i_{k 2}\right) \ldots a\left(i_{1 j}, i_{2 j}, \ldots, i_{k j}\right) . \\
\end{gathered}
$$

If $\left(i_{l 1}, i_{l 2}, \ldots, i_{l j}\right)$ is the ordered $j$ tuple of elements in the range set of $X_{l}$ which sum up to $i_{l}, l=1,2, \ldots, k$.

Setting $s_{1}=s_{2}=\cdots=s_{k}=1$ in 14 , we obtain the joint factorial moment of $\left(N_{1}, N_{2}, \ldots, N_{k}\right)$.

$$
\begin{aligned}
E & {\left[N_{1}\left(N_{1}-1\right) \ldots\left(N_{1}-r_{1}+1\right) \ldots N_{k}\left(N_{k-1}\right) \ldots\left(N_{k}-r_{k+1}\right)\right] } \\
& =e^{-\lambda} \sum_{j=1}^{\infty} \sum_{i_{1} \geq r_{1}, \ldots, i_{k} \geq r_{k}} \frac{\left(\frac{\lambda}{g\left(\theta_{1}, \ldots \theta_{k}\right)}\right)^{j}}{j !} C_{i_{1}, i_{2}, \ldots, i_{k}}(j) i_{1}^{\left(r_{1}\right)} \theta_{1}^{i_{1}} \ldots i_{k}^{\left(r_{k}\right)} \theta_{k}^{i_{k}} .
\end{aligned}
$$

\subsection{Conditional Distribution}

From Johnson et al.(1997), the conditional PGF of $\left(N_{2} \ldots N_{k}\right)$, given $N_{1}$ written

$\Psi_{N_{2} \ldots N_{k} / N_{1}=i_{1}}\left(s_{2}, \ldots, s_{k}\right)$, is

$$
\Psi_{N_{2} \ldots N_{k} / N_{1}=i_{1}}\left(s_{2}, \ldots, s_{k}\right)=\frac{\Psi^{\left(i_{1}, 0, \ldots, 0\right)}\left(0, s_{2}, \ldots, s_{k}\right)}{\Psi^{\left(i_{1}, 0 \ldots, 0\right)}(0,1, \ldots, 1)}
$$

where

$$
\Psi^{\left(i_{1}, 0, \ldots, 0\right)}\left(s_{1}, \ldots, s_{k}\right)=\frac{\partial^{i_{1}} \Psi\left(s_{1}, \ldots, s_{k}\right)}{\partial s_{1}^{i_{1}}}
$$


Substituting $\left(r_{1}, r_{2}, \ldots, r_{k}\right)=\left(i_{1}, 0, \ldots, 0\right)$ and $s_{1}=0$ in $(14)$, we get

$$
\Psi^{\left(i_{1}, 0, \ldots, 0\right)}\left(0, s_{2}, \ldots, s_{k}\right)=e^{-\lambda} \sum_{j=1}^{\infty} \sum_{i_{2}, \ldots, i_{k}} \frac{\left(\frac{\lambda}{g\left(\theta_{1}, \ldots, \theta_{k}\right)}\right)^{j}}{j !} C_{i_{1}, i_{2} \ldots, i_{k}}(j) i_{1} ! \theta_{1}^{i_{1}}\left(\theta_{2} s_{2}\right)^{i_{2}} \ldots\left(\theta_{k} s_{k}\right)^{i_{k}} .
$$

Using (15) and 16 we obtain

$$
\Psi_{N_{2} \ldots N_{k} / N_{1}=i_{1}}\left(s_{2}, \ldots, s_{k}\right)=\frac{\sum_{j=1}^{\infty} \sum_{i_{2}, \ldots, i_{k}} \frac{\left(\frac{\lambda}{g\left(\theta_{1}, \ldots, \theta_{k}\right)}\right)^{j}}{j !} C_{i_{1}, i_{2} \ldots, i_{k}}(j) i_{1} ! \theta_{1}^{i_{1}}\left(\theta_{2} s_{2}\right)^{i_{2}} \ldots\left(\theta_{k} s_{k}\right)^{i_{k}}}{\sum_{j=1}^{\infty} \sum_{i_{2}, \ldots, i_{k}} \frac{\left(\frac{\lambda}{g\left(\theta_{1}, \ldots, \theta_{k}\right)}\right)^{j}}{j !} C_{i_{1}, i_{2} \ldots, i_{k}}(j) i_{1} ! \theta_{1}^{i_{1}} \theta_{2}^{i_{2}} \ldots \theta_{k}^{i_{k}}} .
$$

For $i_{1}=0$, we get

$$
\begin{aligned}
\Psi_{N_{2} \ldots N_{k} / N_{1}=0}\left(s_{2}, \ldots, s_{k}\right) & =\frac{\Psi\left(0, s_{2}, \ldots, s_{k}\right)}{\Psi(0,1, \ldots, 1)} \\
& =e^{-\lambda \frac{g\left(0, \theta_{2}, \ldots, \theta_{k}\right)}{g\left(\theta_{1}, \theta_{2}, \ldots, \theta_{k}\right)}}\left(1-\frac{g\left(0, \theta_{2} s_{2}, \ldots, \theta_{k} s_{k}\right)}{g\left(\theta_{1}, \theta_{2}, \ldots, \theta_{k}\right)}\right) .
\end{aligned}
$$

The conditional PGF of $N_{k}$ given $\left(N_{1}, N_{2}, \cdots, N_{k-1}\right)$ is

$$
\begin{aligned}
\Psi_{N_{k} / N_{1}=i_{1}, \ldots, N_{k-1}=i_{k-1}}\left(s_{k}\right) & =\frac{\Psi^{\left(i_{1}, \ldots, i_{k-1}, 0\right)}\left(0, \ldots, 0, s_{k}\right)}{\Psi^{\left(i_{1}, \ldots, i_{k-1}, 0\right)}(0, \ldots, 0,1)} \\
& =\frac{\sum_{j=1}^{\infty} \sum_{i_{k}} \frac{\left(\frac{\lambda}{g\left(\theta_{1}, \ldots, \theta_{k}\right)}\right)^{j}}{j !} C_{i_{1}, i_{2} \ldots, i_{k}}(j) i_{1} ! i_{2} ! \ldots i_{k} ! \theta_{1}^{i_{1}} \theta_{2}^{i_{2}} \ldots \theta_{k-1}^{i_{k-1}}\left(\theta_{k} s_{k}\right)^{i_{k}}}{\sum_{j=1}^{\infty} \sum_{i_{k}} \frac{\left(\frac{\lambda}{g\left(\theta_{1}, \ldots, \theta_{k}\right)}\right)^{j}}{j !} C_{i_{1}, i_{2} \ldots, i_{k}}(j) i_{1} ! i_{2} ! \ldots i_{k-1} ! \theta_{1}^{i_{1}} \theta_{2}^{i_{2}} \ldots \theta_{k}^{i_{k}}}
\end{aligned}
$$

For $i_{1}=i_{2}=\cdots=i_{k-1}=0$, we get

$$
\begin{aligned}
\Psi_{N_{k} / N_{1}=N_{2}=\cdots=N_{k-1}=0} & =\frac{\Psi\left(0, \ldots, 0, s_{k}\right)}{\Psi(0, \ldots, 0,1)} \\
& =e^{-\lambda \frac{g\left(0, \ldots, 0, \theta_{k}\right)}{g\left(\theta_{1}, \ldots, \theta_{k}\right)}}\left(1-\frac{g\left(0, \ldots, 0, \theta_{k} s_{k}\right)}{g\left(\theta_{1}, \ldots, \theta_{k}\right)}\right)
\end{aligned}
$$

It follows immediately that the conditional mean is

$$
\begin{aligned}
E\left(N_{k} / N_{1}\right. & \left.=i_{1}, N_{2}=i_{2}, \ldots, N_{k-1}=i_{k-1}\right) \\
= & \frac{\sum_{j=1}^{\infty} \sum_{i_{k}} \frac{\left(\frac{\lambda}{g\left(\theta_{1}, \ldots, \theta_{k}\right)}\right)^{j}}{j !} C_{i_{1}, i_{2} \ldots, i_{k}}(j) i_{1} ! \ldots i_{k-1} ! \theta_{1}^{i_{1}} \ldots \theta_{k-1}^{i_{k-1}}\left(\theta_{k} s_{k}\right)^{i_{k}}}{\sum_{j=1}^{\infty} \sum_{i_{k}} \frac{\left(\frac{\lambda}{g\left(\theta_{1}, \ldots, \theta_{k}\right)}\right)^{j}}{j !} C_{i_{1}, i_{2} \ldots, i_{k}}(j) i_{1} ! i_{2} ! \ldots i_{k-1} ! \theta_{1}^{i_{1}}\left(\theta_{2}\right)^{i_{2}} \ldots \theta_{k}^{i_{k}}}
\end{aligned}
$$

In particular

$$
E\left(N_{k} / N_{1}=\cdots=N_{k-1}=0\right)=\frac{\lambda t \theta_{k} \frac{\partial}{\partial \theta_{k}} g\left(0, \ldots, 0, \theta_{k}\right)}{g\left(\theta_{1}, \ldots, \theta_{k}\right)}
$$

\section{Type II bivariate Generalized Power Series Poisson Process}

Consider a compound Poisson process with bivariate generalized power series compounding distribution, given in (1). The resulting bivariate counting process $\left(N_{1}(t), N_{2}(t)\right)$ has type II bivariate generalized power series Poisson 
distribution with parameters $\lambda t, \theta_{1}$ and $\theta_{2}$. i.e,

$$
\begin{aligned}
& f(0,0)=e^{-\lambda t\left(1-\frac{a_{0,0}}{g\left(\theta_{1}, \theta_{2}\right)}\right)}, \\
& f(i, j)=e^{-\lambda t} \theta_{1}^{i} \theta_{2}^{j} \sum_{k=1}^{\infty} \frac{C_{i j}(k)\left(\frac{\lambda}{g\left(\theta_{1}, \theta_{2}\right)}\right)^{k}}{k !}, i, j=0,1, \cdots,(i, j) \neq(0,0),
\end{aligned}
$$

where $C_{i j}(k)=\sum_{\substack{i_{1}+i_{2}+\cdots+i_{k}=i \\ j_{1}+j_{2}+\cdots+j_{k}=j}} a_{i_{1}, j_{1}} a_{l_{2}, j_{2}} \cdots a_{i_{k}, j_{k}}$, if $\left(i_{1}, i_{2}, \cdots, i_{k}\right)$ is the ordered $k$ tuple of elements in the range of $X$ which sums up to $i$ and $\left(j_{1}, j_{2}, \cdots j_{k}\right)$ is the ordered $k$ tuple of elements in the range of $Y$ which sums up to $j$

To express $\left\{\left(N_{1}(t), N_{2}(t)\right), t \geq 0\right\}$ is type II bivariate generalized power series Poisson process with parameters ${ }_{80} \lambda t, \theta_{1}$ and $\theta_{2}$ we use the notation $\left(N_{1}(t), N_{2}(t)\right) \sim B G P S_{I I}\left(\lambda t, \theta_{1}, \theta_{2}\right)$.

Remark: 1. 1.In the case of $g\left(\theta_{1}, \theta_{2}\right)=\left(1-\theta_{1}-\theta_{2}\right)^{-r}, \theta_{1}=\alpha, \theta_{2}=\beta$ and $a(i, j)=\left(\begin{array}{c}i+j \\ j\end{array}\right)\left(\begin{array}{c}r+i+j-1 \\ i+j\end{array}\right)$, the type II bivariate generalized power series Poisson process coincides with bivariate Poisson negative binomial process; see Kostadinova and Minkova(2014).

2.In the case of $g\left(\theta_{1}, \theta_{2}\right)=\left(1-\theta_{1}-\theta_{2}\right)^{-1}, \theta_{1}=\alpha, \theta_{2}=\beta$ and $a(i, j)=\left(\begin{array}{c}i+j \\ j\end{array}\right)\left(\begin{array}{c}r+i+j-1 \\ i+j\end{array}\right)$, the type II bivariate generalized power series Poisson process coincides with type II bivariate Polya-Aeppli process; see Kostadinova(2015).

\section{Type II Bivariate Generalized Power Series Poisson Risk Model}

Let us assume that there are two kinds of claims $X_{1 i}$ and $X_{2 i}$ belonging to two classes. We will investigate a two dimensional model

$$
\begin{aligned}
& U_{1}(t)=u_{1}+c_{1} t-S_{1}(t), \\
& U_{2}(t)=u_{2}+c_{2} t-S_{2}(t),
\end{aligned}
$$

where $u_{i}, i=1,2$, is the initial capital, $c_{i}>0, i=1,2$, is the constant premium income per unit time, $N_{i}(t)$ is the 90 number of claims up to time t, $X_{i k}$ is the size of the $k^{\text {th }}$ claim and $S_{i}(t)=\sum_{j=1}^{N_{i}(t)} X_{i j}, i=1,2$ is the aggregate claims amount for $i^{\text {th }}$ class.

For fixed $i=1,2,\left\{X_{i k}\right\}_{k \geq 1}$ are independent and identically distributed (i.i.d) nonnegative random variable with distribution function $F_{i}\left(X_{i}\right)$ such that $F_{i}(0)=0$ and finite mean $\mu_{i}=E\left(X_{i}\right)$. Assume that $\left\{N_{i}(t), t \geq 0\right\},\left\{\left(X_{1 k}, X_{2 k}\right)\right\}_{k \geq 1}$ are mutually independent and $\left\{\left(X_{1 k}, X_{2 k}\right)\right\}_{k \geq 1}$ is a sequence of i.i.d bivariate random vectors with joint distribution

${ }_{95}$ function $F\left(x_{1}, x_{2}\right)$. Here we assume that bivariate counting process $\left\{\left(N_{1}(t), N_{2}(t)\right), t \geq 0\right\}$ has a type II bivariate generalized power series Poisson process and will call the process the bivariate generalized power series Poisson risk model.

Now we consider the sum of both risk process 17 , the joint capital for the two classes is given by:

$$
U(t)=U_{1}(t)+U_{2}(t)=u+c t-S(t)
$$


where $u=u_{1}+u_{2}, c=c_{1}+c_{2}$ and $S(t)=S_{1}(t)+S_{2}(t)$.

Central problem in risk theory is the modeling of the probability distribution for the aggregate claims. The aggregate claims distribution is mainly used to compute ruin probabilities. Hesselarger(1996) introduced recursive formulas for the joint distribution of the bivariate aggregate claims random variables. Clark and Homer(2003) used Fast Forier Transformation(FFT) to compute bivariate aggregate claims distribution. Here we derive bivariate aggregate claims distribution from type II bivariate generalized power series Poisson risk model via convolution. Let $H\left(u_{1}, u_{2}, t\right)$ denotes the joint cumulative distribution function of bivariate aggregate claims, $\left(S_{1}(t), S_{2}(t)\right)$ and and $F^{* n}(x)$ is the $n$-fold convolution of claim amount distribution which can be calculated recursively as

$$
F^{* n}(x)=\int_{0}^{x} F^{* n-1}(x-y) f(y) d y .
$$

The joint CDF of aggregate claims is given by

$$
\begin{aligned}
H_{\left(S_{1}(t), S_{2}(t)\right)}(x, y, t)= & P\left(S_{1}(t) \leq x, S_{2}(t) \leq y\right) \\
= & \sum_{i, j=0}^{\infty} P\left(N_{1}(t)=i, N_{2}(t)=j\right) F_{1}^{* i}(x) F_{2}^{* j}(y) \\
= & e^{-\lambda t}\left(1-\frac{a_{0,0}}{g\left(\theta_{1}, \theta_{2}\right)}\right) \\
& +e^{-\lambda t} \sum_{i, k=1}^{\infty} \frac{C_{i, 0}(k) \theta_{1}^{i}\left(\frac{\lambda t}{g\left(\theta_{1}, \theta_{2}\right)}\right)^{k}}{k !} F_{1}^{* i}(x) \\
& +e^{-\lambda t} \sum_{j, k=1}^{\infty} \frac{C_{0, j}(k) \theta_{2}^{j}\left(\frac{\lambda t}{g\left(\theta_{1}, \theta_{2}\right)}\right)^{k}}{k !} F_{2}^{* j}(y) \\
& +e^{-\lambda t} \sum_{i, j, k=1}^{\infty} C_{i, j}(k) \theta_{1}^{i} \theta_{2}^{j} \frac{\left(\frac{\lambda t}{g\left(\theta_{1}, \theta_{2}\right)}\right)^{k}}{k !} F_{1}^{* i}(x) F_{2}^{* j}(y) .
\end{aligned}
$$

Let $N(t)=N_{1}(t)+N_{2}(t)$ denotes the total number of claims happened in both classes.

Then the PMF of $N(t)$ is given by

$$
P(N(t)=k)= \begin{cases}e^{-\lambda t}\left(1-\frac{a_{0,0}}{g\left(\theta_{1}, \theta_{2}\right)}\right), & k=0 \\ e^{-\lambda t} \sum_{j=1}^{\infty} \sum_{i=0}^{k} \frac{\left(\frac{\lambda t}{g\left(\theta_{1}, \theta_{2}\right)}\right)^{j}}{j !} C_{i, k-i}(j) \theta_{1}^{i} \theta_{2}^{k-i}, & k=1,2, \cdots\end{cases}
$$

Now we consider the sum of aggregate claims of two classes

$$
S(t)=S_{1}(t)+S_{2}(t)
$$

\section{Case 1:two classes have different claim size distribution}

In this case

$$
S(t)=\sum_{j=1}^{N_{1}(t)} X_{1 j}+\sum_{j=1}^{N_{2}(t)} X_{2 j}
$$


100 and the corresponding $\mathrm{CDF} G(\mathrm{u})$ is given by

$$
\begin{aligned}
G(u)= & P(S(t) \leq x) \\
= & \sum_{i, j=0}^{\infty} P\left(N_{1}(t)=i, N_{2}(t)=j\right) F_{1}^{* i} * F_{2}^{* j}(x) \\
= & e^{-\lambda t\left(1-\frac{a_{0,0}}{g\left(\theta_{1}, \theta_{2}\right)}\right)}+e^{-\lambda t} \sum_{i, k=1}^{\infty} \frac{C_{i, 0}(k) \theta_{1}^{i}\left(\frac{\lambda t}{g\left(\theta_{1}, \theta_{2}\right)}\right)^{k}}{k !} F_{1}^{* i}(x) \\
& +e^{-\lambda t} \sum_{j, k=1}^{\infty} \frac{C_{0, j}(k) \theta_{2}^{j}\left(\frac{\lambda t}{g\left(\theta_{1}, \theta_{2}\right)}\right)^{k}}{k !} F_{2}^{* j}(x)+e^{-\lambda t} \sum_{i, j, k=1}^{\infty} C_{i, j}(k) \theta_{1}^{i} \theta_{2}^{j} \frac{\left(\frac{\lambda t}{g\left(\theta_{1}, \theta_{2}\right)}\right)^{k}}{k !} F_{1}^{* i} * F_{2}^{* j}(x) .
\end{aligned}
$$

\section{Case 2:two classes have the same claim size distribution}

In this case

$$
S(t)=X_{1}+X_{2}+\cdots+X_{N(t)},
$$

where $N(t)=N_{1}(t)+N_{2}(t)$.

Denote by $G(x)$ the CDF of $S(t)$ and is given by

$$
\begin{aligned}
G(x) & =P(S(t) \leq x) \\
& =\sum_{i=0}^{\infty} P(N(t)=i) F^{* i}(x) \\
& =e^{-\lambda t\left(1-\frac{a_{0,0}}{g\left(\theta_{1}, \theta_{2}\right)}\right)}+e^{-\lambda t} \sum_{i, j=1}^{\infty} \sum_{r=0}^{i} \frac{\left(\frac{\lambda t}{g\left(\theta_{1}, \theta_{2}\right)}\right)^{j}}{j !} C_{r, i-r}(j) \theta_{1}^{r} \theta_{2}^{i-r} F^{* i}(x) .
\end{aligned}
$$

\section{Ruin probabilities}

Ruin theory for the bivariate risk model has been extensively considered in the literature. It has been found that ruin probabilities are often fundamental interest in risk management purpose. Chan et al.(2003) discussed various ruin concept for bivariate risk process.

The time of ruin for the $i^{t h}$ class $(i=1,2)$ is defined by

$$
\tau_{i}=\inf \left\{t \geq 0 ; U_{i}(t)<0\right\}
$$

and the corresponding probability of ruin is

$$
\Psi_{i}\left(u_{i}\right)=P\left(\tau_{i}<\infty / U_{i}(0)=u_{i}\right)
$$

If for each $i$, the process $U_{i}(t) \geq 0$ for all $t \geq 0$ (no ruin occurs), we indicae this by writing $\tau_{i}=\infty$. 
Here we consider three kinds of ruin time as follows. The first one is $\tau_{\max }\left(u_{1}, u_{2}\right)=\inf \left\{t \geq 0 / \max \left(U_{1}(t), U_{2}(t)\right)<\right.$ $0\}$, representing the first time when both $U_{1}(t)$ and $U_{2}(t)$ became negative, whereas the second one is $\tau_{\min }\left(u_{1}, u_{2}\right)=$ $\inf \left\{t \geq 0 / \min \left(U_{1}(t), U_{2}(t)\right)<0\right\}$, representing the first time when either $U_{1}(t)$ or $U_{2}(t)$ became negative, and last one is $\tau_{\text {sum }}=\inf \{t \geq 0 / U(t)<0\}$, representing the time when the joint capital for the two classes $U(t)$ became negative. The associated ruin probabilities will be respectively denoted by $\Psi_{\max }\left(u_{1}, u_{2}\right), \Psi_{\min }\left(u_{1}, u_{2}\right)$ and $\Psi_{\text {sum }}\left(u_{1}, u_{2}\right)$. First we derive the expression for the ruin probability $\Psi_{\max }\left(u_{1}, u_{2}\right)$

$$
\begin{aligned}
\Psi_{\max }\left(u_{1}, u_{2}\right) & =P\left(\tau_{\max }<\infty / U_{1}(0)=u_{1}, U_{2}(0)=u_{2}\right) \\
& =P\left(\max \left(U_{1}(t), U_{2}(t)\right)<0\right) \\
& =P\left(U_{1}(t)<0, U_{2}(t)<0\right) \\
& \left.=P\left(S_{1}(t)>u_{1}+c_{1} t, S_{2}(t)\right)>u_{2}+c_{2} t\right) \\
& =\bar{H}\left(u_{1}+c_{1} t, u_{2}+c_{2} t\right) .
\end{aligned}
$$

where $\bar{H}\left(u_{1}, u_{2}\right)$ is the joint survival function of $\left(S_{1}(t), S_{2}(t)\right)$.

Next we consider the expression for the ruin probability $\Psi_{\min }\left(u_{1}, u_{2}\right)$

$$
\begin{aligned}
\Psi_{\min }\left(u_{1}, u_{2}\right) & =P\left(\tau_{\min }<\infty / U_{1}(0)=u_{1}, U_{2}(0)=u_{2}\right) \\
& =P\left(\min \left(U_{1}(t), U_{2}(t)\right)<0\right) \\
& =1-P\left(\min \left(U_{1}(t), U_{2}(t)\right)>0\right) \\
& \left.=1-P\left(U_{1}(t)>0, U_{2}(t)\right)>0\right) \\
& =1-P\left(S_{1}(t)<u_{1}+c_{1} t, S_{2}(t)<u_{2}+c_{2} t\right) \\
& =1-H\left(u_{1}+c_{1} t, u_{2}+c_{2} t\right),
\end{aligned}
$$

where $H\left(u_{1}, u_{2}\right)$ is the joint CDF of $\left(S_{1}(t), S_{2}(t)\right)$ given by 18 .

Finally we derive the expression for the ruin probability $\Psi_{\text {sum }}\left(u_{1}, u_{2}\right)$

$$
\begin{aligned}
\Psi_{\text {sum }}\left(u_{1}, u_{2}\right) & =P\left(\tau_{\text {sum }}<\infty / U_{1}(0)=u_{1}, U_{2}(0)=u_{2}\right) \\
& =P(U(t)<0) \\
& =P(S(t)>u+c t) \\
& =\bar{G}(u+c t)
\end{aligned}
$$

where $\bar{G}(x)$ is the survival function of $S(t)$. 


\subsection{Bounds for Ruin Probability}

Most of the papers in the literature of bivariate risk theory are concerned with ruin probabilities. Exact solutions of these probabilities are rarely available, and existing result are mostly in the form of bounds. Chan et al.(2003), Cai and $\operatorname{Li}(2005)$ and Yuen et al.(2006) derived bounds for the ultimate ruin probability $\Psi_{\min }\left(u_{1}, u_{2}\right)$. Simple bounds for $\Psi_{\max }\left(u_{1}, u_{2}\right)$ was given by Cai and $\operatorname{Li}(2005,2007)$.

The lower and upper bounds on $\Psi_{\max }\left(u_{1}, u_{2}\right)$ and $\Psi_{\min }\left(u_{1}, u_{2}\right)$ are respectively described by the following inequalities.

$$
\begin{array}{r}
\Psi_{1}\left(u_{1}\right) \Psi_{2}\left(u_{2}\right) \leq \Psi_{\max }\left(u_{1}, u_{2}\right) \leq \min \left(\Psi_{1}\left(u_{1}\right), \Psi_{2}\left(u_{2}\right)\right) \\
\max \left(\Psi_{1}\left(u_{1}\right), \Psi_{2}\left(u_{2}\right)\right) \leq \Psi_{\min }\left(u_{1}, u_{2}\right) \leq \Psi_{1}\left(u_{1}\right)+\Psi_{2}\left(u_{2}\right)-\Psi_{1}\left(u_{1}\right) \Psi_{2}\left(u_{2}\right),
\end{array}
$$

115 where the final expression in the second equation is exactly the ruin probability in the case where $\left\{U_{1}(t)\right\}_{t \geq 0}$ and $\left\{U_{2}(t)\right\}_{t \geq 0}$ are independent.

If there is no initial capitals $\left(u_{1}=u_{2}=0\right)$, then the above relations becomes

$$
\begin{array}{r}
\Psi_{1}(0) \Psi_{2}(0) \leq \Psi_{\max }(0,0) \leq \min \left(\Psi_{1}(0), \Psi_{2}(0)\right) \\
\max \left(\Psi_{1}(0), \Psi_{2}(0) \leq \Psi_{\min }(0,0) \leq \Psi_{1}(0)+\Psi_{2}(0)-\Psi_{1}(0) \Psi_{2}(0)\right.
\end{array}
$$

In the case of univariate generalized power series Poisson risk model the ruin probabilities are given by

$$
\Psi_{i}(0)=\frac{\lambda \mu_{i} h_{i}^{\prime}\left(\theta_{i}\right)}{c_{i} h_{i}\left(\theta_{1}\right)} \quad i=1,2 .
$$

Using the equations $(22)$ and $(23)$ we can obtain bounds for the ruin probabilities $\Psi_{\max }(0,0)$ and $\Psi_{\min }(0,0)$ for the type II bivariate generalized power series Poisson risk model and are given by

$$
\begin{gathered}
\frac{\lambda^{2} \mu_{1} \mu_{2} h_{1}^{\prime}\left(\theta_{1}\right) h_{2}^{\prime}\left(\theta_{2}\right)}{c_{1} c_{2} h_{1}\left(\theta_{1}\right) h_{2}\left(\theta_{2}\right)} \leq \Psi_{\max }(0,0) \leq \min \left(\frac{\lambda \mu_{1} h_{1}^{\prime}\left(\theta_{1}\right)}{c_{1} h_{1}\left(\theta_{1}\right)}, \frac{\lambda \mu_{2} h_{2}^{\prime}\left(\theta_{2}\right)}{c_{2} h_{2}\left(\theta_{2}\right)}\right) \\
\max \left(\frac{\lambda \mu_{1} h_{1}^{\prime}\left(\theta_{1}\right)}{c_{1} h_{1}\left(\theta_{1}\right)}, \frac{\lambda \mu_{2} h_{2}^{\prime}\left(\theta_{2}\right)}{c_{2} h_{2}\left(\theta_{2}\right)}\right) \leq \Psi_{\min }(0,0) \leq \frac{\lambda \mu_{1} h_{1}^{\prime}\left(\theta_{1}\right)}{c_{1} h_{1}\left(\theta_{1}\right)}+\frac{\lambda \mu_{2} h_{2}^{\prime}\left(\theta_{2}\right)}{c_{2} h_{2}\left(\theta_{2}\right)}-\frac{\lambda^{2} \mu_{1} \mu_{2} h_{1}^{\prime}\left(\theta_{1}\right) h_{2}^{\prime}\left(\theta_{2}\right)}{c_{1} c_{2} h_{1}\left(\theta_{1}\right) h_{2}\left(\theta_{2}\right)}
\end{gathered}
$$

Moreover, we have

$$
\Psi_{\min }\left(u_{1}, u_{2}\right)=\Psi_{1}\left(u_{1}\right)+\Psi_{2}\left(u_{2}\right)-\Psi_{\max }\left(u_{1}, u_{2}\right) .
$$

In the case of no initial capital above relation is

$$
\Psi_{\min }(0,0)=\Psi_{1}(0)+\Psi_{2}(0)-\Psi_{\max }(0,0) .
$$

and hence,we obtain

$$
\Psi_{\min }(0,0)=\frac{\lambda \mu_{1} h_{1}^{\prime}\left(\theta_{1}\right)}{c_{1} h_{1}\left(\theta_{1}\right)}+\frac{\lambda \mu_{2} h^{\prime}\left(\theta_{2}\right)}{c_{2} h_{2}\left(\theta_{2}\right)}-\Psi_{\max }(0,0)
$$




\section{Two Dimensional Integro Differential Equation}

In this section we will derive partial integro differential equation for the bivariate survival probability for the bivariate surplus process 17 defined in section 6 .

Define the infinite time joint survival probability

$$
\Phi\left(u_{1}, u_{2}\right)=P\left(U_{1}(t) \geq 0, U_{2}(t) \geq 0 ; \text { for all } t \geq 0\right)
$$

and infinite time joint ruin probability is $\Psi\left(u_{1}, u_{2}\right)=1-\Phi\left(u_{1}, u_{2}\right)$.

In a small time interval $(0, h]$, there are following possible cases: no claim, one claim from class 1 and no claim from class 2, no claim from class 1 and one claim from class 2, one or more than one claims from each class. It follows that

$$
\begin{aligned}
\Phi\left(u_{1}, u_{2}\right) & =\left(1-\lambda h\left(1-\frac{a_{0,0}}{g\left(\theta_{1}, \theta_{2}\right)}\right)+o(h)\right) \Phi\left(u_{1}+c_{1} h, u_{2}+c_{2} h\right) \\
& +\left(\frac{a_{10} \theta_{1} h}{g\left(\theta_{1}, \theta_{2}\right)}+o(h)\right) \int_{0}^{u_{1}+c_{1} h} \Phi\left(u_{1}+c_{1} h-x, u_{2}+c_{2} h\right) d F_{1}(x) \\
& +\left(\frac{a_{01} \theta_{2} h}{g\left(\theta_{1}, \theta_{2}\right)}+o(h)\right) \int_{0}^{u_{2}+c_{2} h} \Phi\left(u_{1}+c_{1} h, u_{2}+c_{2} h-y\right) d F_{2}(y) \\
& +\left(\sum_{i, j=1}^{\infty} \frac{a_{i, j} \theta_{1}^{i} \theta_{2}^{j} h}{g\left(\theta_{1}, \theta_{2}\right)}+o(h)\right) \int_{0}^{u_{1}+c_{1} h} \int_{0}^{u_{2}+c_{2} h} \Phi\left(u_{1}+c_{1} h-x, u_{2}+c_{2} h-y\right) d F_{1}^{* i}(x) d F_{2}^{* j}(y),
\end{aligned}
$$

where $F_{i}^{* m}(x), i=1,2 ., m=1,2, \cdots$ is the distribution function of $X_{i 1}+X_{i 2}+\ldots+X_{i m}$.

Rearranging the terms leads to

$$
\begin{aligned}
\frac{\Phi\left(u_{1}+c_{1} h, u_{2}+c_{2} h\right)-\Phi\left(u_{1}, u_{2}\right)}{h} & =\lambda\left(1-\frac{a_{0,0}}{g\left(\theta_{1}, \theta_{2}\right)}\right) \Phi\left(u_{1}+c_{1} h, u_{2}+c_{2} h\right) \\
& +\frac{a_{10} \theta_{1}}{g\left(\theta_{1}, \theta_{2}\right)} \int_{0}^{u_{1}+c_{1} h} \Phi\left(u_{1}+c_{1} h-x, u_{2}+c_{2} h\right) d F_{1}(x) \\
& +\frac{a_{01} \theta_{2}}{g\left(\theta_{1}, \theta_{2}\right)} \int_{0}^{u_{2}+c_{2} h} \Phi\left(u_{1}+c_{1} h, u_{2}+c_{2} h-y\right) d F_{2}(y)+\sum_{i, j=1}^{\infty} \frac{a_{i, j} \theta_{1}^{i} \theta_{2}^{j}}{g\left(\theta_{1}, \theta_{2}\right)} \\
& \int_{0}^{u_{1}+c_{1} h} \int_{0}^{u_{2}+c_{2} h} \Phi\left(u_{1}+c_{1} h-x, u_{2}+c_{2} h-y\right) d F_{1}^{* i}(x) d F_{2}^{* j}(y)+o(h) .
\end{aligned}
$$

As $\mathrm{h}$ tends to zero, we get

$$
\begin{aligned}
c_{1} \frac{\partial}{\partial u_{1}} \Phi\left(u_{1}, u_{2}\right)+c_{2} \frac{\partial}{\partial u_{2}} \Phi\left(u_{1}, u_{2}\right) & =\lambda\left(1-\frac{a_{0,0}}{g\left(\theta_{1}, \theta_{2}\right)}\right) \Phi\left(u_{1}, u_{2}\right) \\
& +\frac{a_{10} \theta_{1}}{g\left(\theta_{1}, \theta_{2}\right)} \int_{0}^{u_{1}} \Phi\left(u_{1}-x, u_{2}\right) d F_{1}(x) \\
& +\frac{a_{01} \theta_{2}}{g\left(\theta_{1}, \theta_{2}\right)} \int_{0}^{u_{2}} \Phi\left(u_{1}, u_{2}-y\right) d F_{2}(y) \\
& +\sum_{i, j=1}^{\infty} \frac{a_{i, j} \theta_{1}^{i} \theta_{2}^{j}}{g\left(\theta_{1}, \theta_{2}\right)} \int_{0}^{u_{1}} \int_{0}^{u_{2}} \Phi\left(u_{1}-x, u_{2}-y\right) d F_{1}^{* i}(x) d F_{2}^{* j}(y)
\end{aligned}
$$

It is difficult to solve this two dimensional integro differential equation. 


\subsection{Laplace Transforms of the Survival Probabilities}

Having obtained the partial integro differential equations(PIDE) for the survival probabilities $\Phi\left(u_{1}, u_{2}\right)$ of the surplus process (17), in the following we will derive the Laplace transforms for the survival probabilities.

Firstly, we define the following Laplace transforms

$\check{\Phi}\left(s_{1}, u_{2}\right)=\int_{0}^{\infty} e^{-s_{1} u_{1}} \Phi\left(u_{1}, u_{2}\right) d u, \tilde{f}_{i}\left(s_{i}\right)=\int_{0}^{\infty} e^{-s_{i} x} d F_{i}(x), i=1,2$. and

$\check{\Phi}\left(s_{1}, s_{2}\right)=\int_{0}^{\infty} \int_{0}^{\infty} e^{-s_{1} u_{1}-s_{2} u_{2}} \Phi\left(u_{1}, u_{2}\right) d u$.

Taking Laplace transform on both sides of the PIDE (24) with respect to $u_{1}$, we get

$$
\begin{aligned}
c_{1}\left(s_{1} \check{\Phi}\left(s_{1}, u_{2}\right)-\Phi\left(0, u_{2}\right)\right)+c_{2} \frac{\partial}{\partial u_{2}} \check{\Phi}\left(s_{1}, u_{2}\right) & =\lambda\left(1-\frac{a_{0,0}}{g\left(\theta_{1}, \theta_{2}\right)}\right) \check{\Phi}\left(s_{1}, u_{2}\right)+\frac{a_{10} \theta_{1} \tilde{f}_{1}\left(s_{1}\right)}{g\left(\theta_{1}, \theta_{2}\right)} \check{\Phi}\left(s_{1}, u_{2}\right) \\
& +\frac{a_{01} \theta_{2}}{g\left(\theta_{1}, \theta_{2}\right)} \int_{0}^{u_{2}} \check{\Phi}\left(s_{1}, u_{2}-y\right) d F_{2}(y) \\
& +\sum_{i, j=1}^{\infty} \frac{a_{i, j} \theta_{1}^{i} \theta_{2}^{j} \tilde{f}_{1}^{* i}\left(s_{1}\right)}{g\left(\theta_{1}, \theta_{2}\right)} \int_{0}^{u_{2}} \check{\Phi}\left(s_{1}, u_{2}-y\right) d F_{2}^{* j}(y),
\end{aligned}
$$

On simplification we get

$$
\begin{aligned}
c_{2} \frac{\partial}{\partial u_{2}} \check{\Phi}\left(s_{1}, u_{2}\right) & =c_{1} \Phi\left(0, u_{2}\right)+\left(\lambda\left(1-\frac{a_{0,0}}{g\left(\theta_{1}, \theta_{2}\right)}\right)-c_{1} s_{1}+\frac{a_{10} \theta_{1} \tilde{f}_{1}\left(s_{1}\right)}{g\left(\theta_{1}, \theta_{2}\right)}\right) \check{\Phi}\left(s_{1}, u_{2}\right) \\
& +\frac{a_{01} \theta_{2}}{g\left(\theta_{1}, \theta_{2}\right)} \int_{0}^{u_{2}} \check{\Phi}\left(s_{1}, u_{2}-y\right) d F_{2}(y)+\sum_{i, j=1}^{\infty} \frac{a_{i, j} \theta_{1}^{i} \theta_{2}^{j} \tilde{f}_{1}^{* i}\left(s_{1}\right)}{g\left(\theta_{1}, \theta_{2}\right)} \int_{0}^{u_{2}} \check{\Phi}\left(s_{1}, u_{2}-y\right) d F_{2}^{* j}(y),
\end{aligned}
$$

Taking Laplace transform on both sides of the PIDE 25 with respect to $u_{2}$, we get

$$
\begin{aligned}
c_{2}\left(s_{2} \check{\Phi}\left(s_{1}, s_{2}\right)-\check{\Phi}\left(s_{1}, 0\right)\right) & =c_{1} \check{\Phi}\left(0, s_{2}\right)+\left(\lambda\left(1-\frac{a_{0,0}}{g\left(\theta_{1}, \theta_{2}\right)}\right)-c_{1} s_{1}+\frac{a_{10} \theta_{1} \tilde{f}_{1}\left(s_{1}\right)}{g\left(\theta_{1}, \theta_{2}\right)}\right) \check{\Phi}\left(s_{1}, s_{2}\right) \\
& +\frac{a_{01} \theta_{2} \tilde{f}_{2}\left(s_{2}\right)}{g\left(\theta_{1}, \theta_{2}\right)} \check{\Phi}\left(s_{1}, s_{2}\right)+\sum_{i, j=1}^{\infty} \frac{a_{i, j} \theta_{1}^{i} \theta_{2}^{j} \tilde{f}_{1}^{* i}\left(s_{1}\right) \tilde{f}_{2}^{* j}\left(s_{2}\right)}{g\left(\theta_{1}, \theta_{2}\right)} \check{\Phi}\left(s_{1}, s_{2}\right)
\end{aligned}
$$

Finally we get

$$
\check{\check{\Phi}}\left(s_{1}, s_{2}\right)=\frac{c_{1} s_{1} \check{\Phi}\left(0, s_{2}\right)+c_{2} s_{2} \check{\Phi}\left(s_{1}, 0\right)}{c_{1} s_{1}+c_{2} s_{2}-\lambda\left(1-\frac{a_{0,0}}{g\left(\theta_{1}, \theta_{2}\right)}\right)-\frac{a_{10} \theta_{1} \tilde{f}_{1}\left(s_{1}\right)}{g\left(\theta_{1}, \theta_{2}\right)}-\frac{a_{01} \theta_{2} \tilde{f}_{2}\left(s_{2}\right)}{g\left(\theta_{1}, \theta_{2}\right)}-\sum_{i, j=1}^{\infty} \frac{a_{i, j} \theta_{1}^{i} \theta_{2}^{j} \tilde{f}_{1}^{* i}\left(s_{1}\right) \tilde{f}_{2}^{* j}\left(s_{2}\right)}{g\left(\theta_{1}, \theta_{2}\right)}}
$$

\section{Conclusion}

In this paper we introduced the type II bivariate generalized power series Poisson distribution as a compound Poisson distribution with generalized power series compounding distribution. We have considered the bivariate risk model with type II bivariate generalized power series Poisson distribution as claim number distribution. Three models of ruin and the probabilities of ruin for the type II bivariate generalized power series Poisson risk model are investigated. Also the bounds for ruin probabilities are developed. We obtained PIDE for the survival probability and derived an expression for bivariate Laplace transform of ruin probabilities. 


\section{Acknowledgments}

The authors acknowledge the financial support from UGC, Government of India towards this research.

\section{References}

Johnson N.L, Kemp A.W. and Kotz S. (2005): Univariate Discrete Distributions, Wiley Series in Probability and Mathematical Statistics, third edition.

Johnson N.L, Kotz S. and Balakrishnan N.(1997): Discrete Multivariate Distributions, John Wiley and Sons, New York.

Omey E. and Minkova L.D.(2013): Bivariate Geometric Distributions, Hub Research Papers Economics and Business Science.

Dufresne F.and Gerber H.U.(1989): Three methods to calculate the probability of ruin, ASTIN Bulletin, 19(1): 71-90.

Clark D.R. and Homer D.L.(2003): Insurance Applications of Bivariate Distributions, Proceedings of the Casuality Actuarial Society XC, 274-30\%.

Tongling L., Junyi G. and Xin Z.(2011): Some Results on Bivariate Compound Poisson Risk Model, Chinese Journal of Applied Probability and Statistics, vol.27 No.5.

Kocherlakota S. and Kocherlakota K.(1992): Bivariate discrete distributions, Marcel Dekker, New York.

Patil G.P.(1962): Certain properties of the generalized power series distribution, Annals of the Institute of Statistical Mathematics 14:179-182.

Chan W.S, Yang H. and Zhang L.(2003): Some results on ruin probabilities in a two dimensional risk model, Insurance Mathematics and Economics, 33, 345-358.

Yuen K.C, Guo J. and Wu X.(2006): On the first time of ruin in the bivariate compound poisson model, Mathematics and Economics, 38: 298-308.

Cai J. and Li H.(2005): Multivariate risk model of phase type, Insurance: Mathematics and Economics, 36: 137-152.

Cai J. and Li H.(2007): Dependence properties and bounds for ruin probabilities in multivariate compound risk models, Journal of Multivariate Analysis, 98: 757-773.

Minkova L.D and Balakrishnan N.(2014):Type II bivariate Polya-Aeppli distribution, Statistics and Probability Letters, 88: 40-49.

Kostadinova K. and Minkova L.(2014): On a Bivariate Poisson Negative Binomial Risk Process, Biomath 
Kostadinova K.(2015):Bivariate Polya-Aeppli risk model, Tom 54,6.1.

(C) 2016 by the authors; licensee Preprints, Basel, Switzerland. This article is an open access article distributed under the terms and conditions of the Creative Commons by Attribution (CC-BY) license (http://creativecommons.org/licenses/by/4.0/). 